\title{
Contenidos escolares en ciencias naturales desde el currículo oficial de Colombia
}

\author{
School Contents on Natural Sciences from the Official Curriculum of Colombia
}

\section{Conteúdos escolares em ciências naturais do currículo oficial da Colômbia}

\author{
Ana-María Cárdenas-Navas ${ }^{1}$ \\ Carmen-Alicia Martínez-Rivera ${ }^{2}$
}

Recibido: febrero de 2021

Aceptado: junio de 2021

Para citar este artículo: Cárdenas-Navas, A. M., y Martínez-Rivera, C. A. (2021). Contenidos escolares en ciencias naturales desde el currículo oficial de Colombia. Revista Científica, 42(3), 328-338. https://doi. org/10.14483/23448350.17614

\section{Resumen}

Este artículo da cuenta de los contenidos escolares (tipos y niveles de organización) que subyacen en las orientaciones curriculares expedidas por el Ministerio de Educación Nacional de Colombia para el área de Ciencias Naturales, estudio que se adelantó en el Doctorado Interinstitucional en Educación. La investigación se enmarca en el enfoque cualitativo, desde una perspectiva interpretativa y asume como método el estudio de caso. Se examinaron los Lineamientos Curriculares, los Estándares Básicos de Competencias y los Derechos Básicos del Aprendizaje, y se realizó una entrevista semiestructurada a un coautor por cada orientación. Los datos fueron tratados a través del análisis de contenido, con apoyo del software Atlas.ti. Se destacan como resultados los diferentes tipos de contenidos escolares conceptuales, procedimentales y actitudinales, y se evidencian algunas articulaciones entre dichos contenidos y las propuestas de organización, como la formulación de niveles de complejidad y los ejes articuladores. Se concluye que los tipos de contenidos escolares están vinculados de manera directa con una visión disciplinar (nivel instruccional-cientificista); en cambio, para la organización de los contenidos escolares se propone una construcción pedagógica y didáctica propia (nivel integrador-transformador).

Palabras clave: ciencias naturales; Colombia; conocimiento escolar; contenidos escolares; orientaciones curriculares.

\begin{abstract}
This article offers an account of the school contents (types and levels of organization) that underlie the curricular orientations issued by the Colombian Ministry of Education for the Natural Sciences area. This study was carried out in the Interinstitutional Doctorate in Education. This research is based on the qualitative approach from an interpretative perspective under the study case methodology. The
\end{abstract}

1. Universidad Distrital Francisco José de Caldas, Bogotá D.C., Colombia.

2. Ph. D. Universidad Distrital Francisco José de Caldas, Bogotá D.C., Colombia. camartinezr@udistrital.edu.co 
Curricular Guidelines (Lineamientos Curriculares), the Core Competency Standards (Estándares Básicos de Competencias), and the Basic Learning Rights (Derechos Básicos del Aprendizaje) were examined, and a semistructured interview was performed with one co-author of each orientation. All the data were examined through content analysis supported by the Atlas.ti software. Different types of conceptual, procedural, and attitudinal school contents stand out as results, and some articulations between said contents and the organization proposals are evidenced, such as the devising of levels of complexity and articulating axes. It is concluded that the types of school contents are directly related to a disciplinary point of view (instructional-scientist level), whereas, for the organization of such contents, a pedagogical construction and a particular didactic (integrative-transformative level) is proposed.

Keywords: Colombia; curricular guidelines; natural sciences; school content; school knowledge.

\section{Resumo}

Este artigo procura dar conta dos conteúdos escolares (tipos e níveis de organização) que fundamentam as orientações curriculares emitidas pelo Ministério da Educação Nacional da Colômbia para a área de Ciências Naturais, estudo realizado no Doutorado Interinstitucional em Educação. Foram analisadas as Diretrizes Curriculares, Normas Básicas de Competência e Direitos Básicos de Aprendizagem e foe foi complementado com uma entrevista com os coautores, $\mathrm{O}$ software Atlas.ti foi usado para o tratamento dos dados. Destacam-se como resultados os diferentes tipos de conteúdos escolares conceituais, procedimentais e atitudinais, evidenciando-se algumas articulações entre esses conteúdos e as propostas organizacionais, como a formulação de níveis de complexidade e eixos articulatórios. Conclui-se que os tipos de conteúdos escolares estão diretamente ligados a uma visão disciplinar (nível científico-instrucional), por outro lado, para a organização dos conteúdos escolares, uma construção pedagógica e didática própria (nível integrativo-transformador) é proposto.

Palavras-chaves: Ciências naturais, conhecimento escolar, conteúdos escolares, orientações curriculares, Colômbia.

\section{Introducción}

El presente artículo da cuenta de los resultados de la macro categoría "contenidos escolares" (tipos y niveles de organización), en el marco de la tesis doctoral que indaga por el conocimiento escolar (CE) en ciencias, que se propone en los Lineamientos Curriculares (LCNEA), los Estándares Básicos de Competencias (ECN) y los Derechos Básicos del Aprendizaje (DCN) para el área de Ciencias Naturales, expedidos por el Ministerio de Educación Nacional de Colombia (MEN). En la tabla 1, para cada orientación, se ilustra la normatividad que sustenta su expedición, y el plan de gobierno o plan sectorial del periodo en el que se elaboró.

Tabla 1. Soporte normativo y periodo de expedición de las orientaciones curriculares analizadas

\begin{tabular}{|c|c|c|}
\hline $\begin{array}{l}\text { Orientación } \\
\text { curricular }\end{array}$ & Normatividad & Plan de gobierno o plan sectorial \\
\hline LCNEA (MEN, 1998) & $\begin{array}{l}\text { Ley General de Educación (Ley 115/94) } \\
\text { (Artículo 78) }\end{array}$ & $\begin{array}{c}\text { Plan Nacional de Desarrollo e Inversiones } \\
\text { (1995-1998). "El salto social" }\end{array}$ \\
\hline ECN (MEN, 2006) & Ley 715 de 2001 (Artículo 5) & $\begin{array}{l}\text { Plan de Desarrollo Educativo (2002-2006) } \\
\text { "La revolución educativa" }\end{array}$ \\
\hline DCN (MEN, 2016) & & $\begin{array}{l}\text { Plan sectorial 2010-2014 "Educación de } \\
\text { calidad - El camino para la prosperidad" } \\
\text { Plan Nacional de Desarrollo 2014-2018 } \\
\text { "Colombia, la mejor educada" }\end{array}$ \\
\hline
\end{tabular}

Fuente: Elaboración propia. 
Como se aprecia en la tabla los LCNEA y los ECN tienen como base, para el primer caso, la Ley General de Educación y, para el segundo caso, la Ley 715 de 2001, por lo que se consideran referentes de calidad. Para el caso de los DCN no hay una normativa específica, sino que estos se construyen a partir de lo planteado en los planes sectoriales de educación, o planes de gobierno, dos para este caso: el Plan sectorial 2010-2014 y el Plan Nacional de Desarrollo 2014-2018, por lo anterior los DCN se definen como referentes de actualización curricular.

\section{El qué de la formación en ciencias naturales}

Entre los asuntos de interés en el análisis de los currículos oficiales están el "qué enseñar" y el "cómo se organiza el qué enseñar", ya que según lo plantea Goodson (1991) es importante en el estudio de las disciplinas o materias escolares dar cuenta de la relación de estas con los contenidos y con los procesos en el contexto escolar, o desde el análisis de lo que implica la educación escolar. Al respecto, Mauri (1993) plantea que abordar los contenidos escolares es un asunto de gran complejidad que da cuenta del qué se selecciona:

A simple vista, hablar de los contenidos escolares puede parecer abordar un tema fácil, por lo conocido que resulta para todos aquellos que nos dedicamos a la educación. Sin embargo, lejos de esta primera sensación de simplicidad, afrontar este tema supone intentar resolver un problema en el que se hallan implicados todos los elementos más característicos de la educación escolar: para qué se enseña, qué se selecciona, con qué medios se enseña, etc. La relación de todos estos aspectos no ha sido resuelta siempre de la misma manera, por lo menos en las últimas décadas, en la historia más reciente de la educación escolar. (p. 1)

En el marco de la didáctica de las ciencias, y específicamente desde la visión compleja del conocimiento escolar, García (1998) indica la importancia de dar cuenta del sistema de ideas (qué enseñar), que implica unos tipos de contenidos, una forma de organización, de selección y de complejización del conocimiento cotidiano, esta última idea reconocida además en planteamientos como los de Merchán y García (1997):

Se trataría de enriquecer el conocimiento de los sujetos con una visión más compleja del mundo, trabajando en el aula un tipo de conocimiento - el conocimiento escolar- que, originado en la integración didáctica de diferentes formas de saber (científico, ideológico-filosófico, cotidiano, artístico, etc.), suponga una construcción crítica y una mejora de conocimiento cotidiano que capacite a los individuos para una participación más consciente en la gestión y resolución de problemas socioambientales propios de nuestro mundo. (p. 9)

Como se puede apreciar en la reflexión de Merchán y García (1997), para los contenidos escolares se destacan tanto la selección, formulación y organización, como la importancia que recobran los objetos de estudio del contexto para integrar conceptos, destrezas, valores e interpretaciones, entre otras.

También como lo explicita Martínez (2017), reconociendo el recorrido de las investigaciones del conocimiento profesional de los profesores de ciencias y el conocimiento escolar (Martínez, 2000; Martínez, 2005; Martínez y Valbuena, 2013), uno de los asuntos analizados responde a la pregunta ¿qué enseñar?, esto en el contexto nacional se ha venido rastreando desde el trabajo de Martínez, Lopes, Cárdenas y Jirón, (2016), en el que se analizan orientaciones para el área de Ciencias Naturales en una de las secretarías de educación, dando cuenta de los tipos y los niveles de organización de los contenidos escolares, entre otras categorías, en el marco de la caracterización del conocimiento escolar.

\section{Referentes metodológicos}

La investigación se enmarca en la didáctica de las ciencias, desde un enfoque cualitativo que, como lo propone Vasilachis (2006), retomando a Mason (1996), se centra en las siguientes características: 
a) Fundada en una posición filosófica ampliamente interpretativa en el sentido que se interesa en las formas en las que el mundo social es interpretado, comprendido, experimentado y producido; b) basada en métodos de generación de datos flexibles y sensibles al contexto social en el que se producen, y c) sostenida por métodos de análisis y explicación que abarcan la comprensión de la complejidad, el detalle y el contexto. (p. 25)

Igualmente, desde la perspectiva interpretativa el investigador "privilegia lo profundo sobre lo superficial, lo intenso sobre lo extenso, lo particular sobre las generalidades, la captación del significado y del sentido interno subjetivo, antes que la observación exterior de presuntas regularidades objetivas" (Vasilachis 2006, p. 49).

Desde esta perspectiva, en el marco de la tesis doctoral, se definió como método el estudio de caso, y como técnicas, el análisis de documentos y las entrevistas semiestructuradas a expertos.

Teniendo en cuenta lo planteado por Stake (1999), la caracterización de las orientaciones curriculares de ciencias naturales expedidas por el MEN se constituye en un estudio de caso único de carácter intrínseco, pues el caso está conformado por los documentos preseleccionados inicialmente por su carácter de orientación curricular para el área de Ciencias Naturales (LCNEA, ECN y DCN). El interés intrínseco está en dar cuenta del conocimiento escolar, tanto desde lo particular, como desde la relación con las orientaciones curriculares en mención, con el propósito de aportar a una de las necesidades identificadas en ese campo del conocimiento escolar.

Como ya se indicó, se realizaron entrevistas semiestructuradas que, como lo menciona Flick (2012), en el campo de la investigación cualitativa han suscitado gran interés y relevancia porque dan la posibilidad al entrevistado de poder expresar sus puntos de vista frente a la situación abordada, por medio de un conjunto de preguntas relativamente abiertas. Dentro de las entrevistas semiestructuradas se eligió la entrevista a expertos, ya que centra su atención en la calidad de experto en un cierto campo de dominio para analizar y comparar el conocimiento del mismo, por lo que para cada orientación se llevó a cabo una entrevista a uno de los coautores.

La entrevista semiestructurada tuvo como propósito general ampliar, corroborar o profundizar en aspectos que aparecen consignados en los documentos y que se identificaron como relevantes en este proceso de caracterización del conocimiento escolar en ciencias naturales, el cual subyace en las orientaciones analizadas.

La codificación se llevó a cabo con apoyo del software Atlas.ti por sus bondades relacionadas con la construcción de unidades o segmentos asociados a palabras clave o códigos que se pueden mostrar de diferentes formas (Chernobilsky, 2006). Con base en el análisis textual discursivo, teniendo en cuenta los planteamientos de Moraes y Galiazzi (2006) y las recomendaciones de trabajos anteriores (Martínez, 2000; Martínez y Valbuena, 2013; Martínez, 2016), desde el análisis de contenido (Bardín, 1996), se desarrolló un proceso de codificación, elaboración de unidades de información, categorización, inferencias y establecimiento de relaciones.

Las subcategorías que refieren a la macro categoría "contenidos escolares" (tipos y niveles de organización) tienen en cuenta, por una parte, los contenidos escolares que se retoman de investigaciones en el marco del conocimiento profesional de los profesores de ciencias sobre el conocimiento escolar (Martínez, 2000; Martínez, 2016; Martínez y Valbuena, 2013; Martínez et al., 2016) y, por otra parte, las subcategorías emergentes que subyacen al proceso de codificación y análisis de las orientaciones curriculares. Es importante mencionar que el proceso de codificación fue triangulado por el equipo de investigadoras a partir de citas asignadas como "dudosas".

Para la macro categoría "contenidos escolares" (tipos y niveles de organización) se proponen niveles de complejidad (ver tabla 2 para los tipos de contenidos y tabla 3 que presenta los niveles de organización), a partir del constructo teórico y metodológico denominado Hipótesis de Progresión (HdP) (García, 1998; Martínez y Martínez, 2012) 
o Hipótesis de Progresión-Transición (HdP-T) del conocimiento profesional del profesor de ciencias sobre el conocimiento escolar, que tiene su base en trabajos como el de Martínez (2000), a partir de su tesis doctoral, y Martínez y Valbuena, 2013 y Martínez (2016). Como se menciona en estos trabajos, este constructo plantea la existencia desde un nivel inicial (visión simple), hasta un nivel de referencia (visión compleja) flexible, con ciclos y fluctuaciones propios del proceso.

Tabla 2. Niveles de complejidad. Subcategorías referidas a los tipos de contenidos escolares del conocimiento escolar en ciencias naturales

\begin{tabular}{cc}
\hline \multicolumn{2}{c}{ Nivel 2A. Instruccional/cientificista-NIC } \\
\hline 2A1.NIC & 2A2.NIC \\
\hline $\begin{array}{c}\text { Contenidos conceptuales, procedimentales o } \\
\text { el conocimiento científico, las formas de proceder de } \\
\text { la comunidad científica y las actitudes científicas }\end{array}$ & $\begin{array}{c}\text { Integración de contenidos conceptuales, procedimentales } \\
\text { o actitudinales en los que la base son los conceptos del } \\
\text { conocimiento científico, el proceder de la comunidad } \\
\text { científica y el desarrollo de las actitudes científicas }\end{array}$ \\
\hline
\end{tabular}

\begin{tabular}{|c|c|c|c|}
\hline \multicolumn{4}{|c|}{ Nivel 3. Integrador/Transformador-NIT } \\
\hline 3A1.NIT & 3B1.NIT & 3C1.NIT & 3C2.NIT \\
\hline $\begin{array}{l}\text { Integración de contenidos } \\
\text { conceptuales, procedimentales o } \\
\text { actitudinales que aluden a conceptos } \\
\text { de las ciencias, pero las prácticas } \\
\text { (procedimentales) están asociadas } \\
\text { al contexto cotidiano o local y } \\
\text { buscan el desarrollo de compromisos } \\
\text { personales o sociales (actitudinales) }\end{array}$ & $\begin{array}{c}\text { Integraciones de contenidos } \\
\text { conceptuales, procedimentales } \\
\text { o actitudinales que aportan } \\
\text { al análisis de situaciones o } \\
\text { problemáticas, a la importancia de } \\
\text { los estados de bienestar personal, } \\
\text { social y cultural y a la búsqueda } \\
\text { de cambios de actitud y prácticas } \\
\text { personales y colectivas }\end{array}$ & $\begin{array}{l}\text { Integración de contenidos } \\
\text { procedimentales y actitudinales } \\
\text { que proponen una práctica } \\
\text { o acción en relación con } \\
\text { compromisos, valores o } \\
\text { actitudes para la vida personal, } \\
\text { para la convivencia o para } \\
\text { aportar a las relaciones ser } \\
\text { humano-naturaleza }\end{array}$ & $\begin{array}{c}\text { Integración de contenidos } \\
\text { conceptuales, procedimentales } \\
\text { y actitudinales que abordan } \\
\text { situaciones o problemáticas } \\
\text { naturales o sociales } \\
\text { que incluyen referentes } \\
\text { disciplinares, contextuales, } \\
\text { sociales y culturales }\end{array}$ \\
\hline
\end{tabular}

Fuente: elaboración propia.

Tabla 3. Niveles de complejidad. Subcategorías referidas a los niveles de organización de los contenidos escolares del conocimiento escolar en ciencias naturales

\begin{tabular}{|c|c|c|c|c|c|}
\hline \multicolumn{6}{|c|}{ Nivel 3. Integrador/Transformador-NIT } \\
\hline \multicolumn{2}{|l|}{ 3A1.NIT } & \multicolumn{2}{|l|}{ 3A2.NIT } & 3B1.NIT & 3B2.NIT \\
\hline \multicolumn{2}{|c|}{$\begin{array}{l}\text { Progresiones de aprendizajes en } \\
\text { los que se establecen cuáles son } \\
\text { los contenidos escolares que se } \\
\text { han transitado en el grado anterior } \\
\text { o que se conectan con algún grado } \\
\text { anterior, cuáles se quieren abordar } \\
\text { en el presente grado y cuáles } \\
\text { para el grado subsiguiente, desde } \\
\text { criterios pedagógicos o didácticos }\end{array}$} & \multicolumn{2}{|c|}{$\begin{array}{l}\text { Niveles de complejidad en } \\
\text { espiral que dan cuenta de } \\
\text { las improntas de los niveles } \\
\text { educativos y de la complejidad } \\
\text { de los contenidos escolares } \\
\text { por bloques o conjuntos } \\
\text { de grados que tienen como } \\
\text { característica la integralidad y } \\
\text { la interdisciplinariedad }\end{array}$} & $\begin{array}{l}\text { Procesos que posibilitan } \\
\text { la articulación de saberes, } \\
\text { conocimientos, habilidades, } \\
\text { actitudes, y que implican acciones de } \\
\text { integración, profundización, reflexión } \\
\text { para la construcción no solo de } \\
\text { conocimientos, sino el desarrollo de } \\
\text { pensamiento crítico y analítico }\end{array}$ & $\begin{array}{l}\text { Estrategias pedagógicas o } \\
\text { metodológicas centradas en la } \\
\text { posibilidad de integración de } \\
\text { diferentes tipos de contenidos } \\
\text { (ej. proyectos pedagógicos; } \\
\text { mallas de aprendizaje; } \\
\text { situaciones problema, etc.) }\end{array}$ \\
\hline \multicolumn{3}{|l|}{ Nivel 1. Tradicional-NT } & \multicolumn{2}{|c|}{ Nivel 2A. Instruccional/Cientificista-NIC } & \\
\hline 1.NT & & 2A1.NIC & & 2A2.NIC & 2A3.NIC \\
\hline $\begin{array}{l}\text { Visión simple de } \\
\text { organización. } \\
\text { Listado de contenidos } \\
\text { (a modo de listado de } \\
\text { temas) }\end{array}$ & $\begin{array}{l}\text { Mapas d } \\
\text { por ex } \\
\text { diálogo } \\
\text { como el } \\
\text { aprendiz } \\
\text { acciones } \\
\text { de las }\end{array}$ & $\begin{array}{l}\text { e relaciones elaborados } \\
\text { upertos que ponen en } \\
\text { elementos articuladores } \\
\text { grado, los entornos, los } \\
\text { ajes estructurantes y las } \\
\text { asociadas a aprendizajes } \\
\text { iencias (instruccional) }\end{array}$ & $\begin{array}{r}\text { Con } \\
\text { un } \\
\text { difere } \\
\text { mov } \\
\text { La ba } \\
\text { cie } \\
\text { comu }\end{array}$ & $\begin{array}{l}\text { nceptos estructurantes concebidos como } \\
\text { nidades básicas para el aprendizaje en } \\
\text { entes contextos y que posibilitan además } \\
\text { vilizar otros saberes, actitudes, prácticas. } \\
\text { se de estos conceptos es el conocimiento } \\
\text { entífico, las maneras de proceder de la } \\
\text { unidad científica y las actitudes científicas }\end{array}$ & $\begin{array}{c}\text { Ejes articuladores en los que } \\
\text { se identifica la integración } \\
\text { entre diferentes tipos de } \\
\text { contenidos (aunque incluyen } \\
\text { contenidos contextuales, se } \\
\text { da prelación a contenidos } \\
\text { propios de las ciencias) }\end{array}$ \\
\hline
\end{tabular}

Fuente: elaboración propia. 
Tabla 4. Documentos analizados en la unidad hermenéutica-UH

\begin{tabular}{|c|c|c|c|c|}
\hline $\begin{array}{l}\text { Orden } \\
\text { dado en } \\
\text { la UH }\end{array}$ & $\begin{array}{l}\text { Nombre del documento de la orientación } \\
\text { o de la entrevista }\end{array}$ & Sigla & $\begin{array}{l}\text { Designación del documento o } \\
\text { entrevista para presentación } \\
\text { de los resultados }\end{array}$ & $\begin{array}{l}\text { Ejemplo del identificador numérico de las } \\
\qquad \text { citas }^{3}\end{array}$ \\
\hline P1 & $\begin{array}{l}\text { Lineamientos Curriculares - Ciencias } \\
\text { Naturales y Educación Ambiental }\end{array}$ & LCNEA & D1 & $\begin{array}{l}\text { 1:310 [El artículo } 67 \text { establece que...] } \\
(27: 3470-27: 3740)\end{array}$ \\
\hline P2 & $\begin{array}{l}\text { Cuadro anexo. Lineamientos Curriculares - } \\
\text { Ciencias Naturales y Educación Ambiental }\end{array}$ & LCNEA & D2 & $\begin{array}{l}2: 75 \text { [Observar que las características...] } \\
(1: 2860-1: 3022)\end{array}$ \\
\hline P3 & $\begin{array}{l}\text { Estándares Básicos de Competencias en } \\
\text { Ciencias Naturales }\end{array}$ & $\mathrm{ECN}$ & D3 & $\begin{array}{l}\text { 3:192 [Teniendo en consideración...] } \\
(28: 1446-28: 2045)\end{array}$ \\
\hline P4 & $\begin{array}{l}\text { Derechos Básicos del Aprendizaje. } \\
\text { Fundamentación teórica }\end{array}$ & $\mathrm{DCN}$ & D4 & $\begin{array}{l}\text { 4:55 [Entonces el rango que se busca...] } \\
(23: 720-23: 1305)\end{array}$ \\
\hline P5 & $\begin{array}{l}\text { Derechos Básicos de Aprendizaje. DBA de } \\
\text { grado } 1^{\circ} \text { a grado } 11^{\circ}\end{array}$ & $\mathrm{DCN}$ & D5 & $\begin{array}{l}\text { 5:170 [Construye experimentalmente ci...] } \\
(19: 253-19: 1041)\end{array}$ \\
\hline P6 & $\begin{array}{l}\text { Derechos Básicos de Aprendizaje. } \\
\text { Documento para la implementación de los } \\
\text { DBA. Mallas de aprendizaje. Presentación } \\
\text { general y por áreas. Ejemplo de Malla de } \\
\text { aprendizaje (grado } 5^{\circ} \text { ) }\end{array}$ & $\mathrm{DCN}$ & D6 & $\begin{array}{l}\text { 6:101 [Para ello se deben diseñar act...] } \\
(25: 478-25: 631)\end{array}$ \\
\hline P7 & $\begin{array}{l}\text { Entrevista coautora. Lineamientos } \\
\text { Curriculares - Ciencias Naturales y } \\
\text { Educación Ambiental. }\end{array}$ & $\begin{array}{l}\text { Entrevista } \\
\text { LCNEA }\end{array}$ & ENT-LCNEA & $\begin{array}{l}\text { P 7: Entrevista.LCNEA.UH1.pdf - 7:131 } \\
\text { [Voy a resumir así...] (27:2787-27:3072) }\end{array}$ \\
\hline P8 & $\begin{array}{l}\text { Entrevista coautor. Estándares Básicos de } \\
\text { Competencias en Ciencias Naturales y } \\
\text { Sociales. }\end{array}$ & $\begin{array}{l}\text { Entrevista } \\
\text { ECN }\end{array}$ & ENT-ECN & $\begin{array}{l}\text { P 8: Entrevista_ECN.UH1.pdf - 8:57 } \\
\text { [Creemos que enseñar Ciencias...] } \\
(10: 3124-10: 3634)\end{array}$ \\
\hline P9 & $\begin{array}{l}\text { Entrevista coautora. Derechos Básicos del } \\
\text { Aprendizaje. Ciencias Naturales. }\end{array}$ & $\begin{array}{l}\text { Entrevista } \\
\text { DCN }\end{array}$ & ENT-DCN & $\begin{array}{l}\text { P 9: Entrevista_DCN.UH1.pdf - 9:4 [Otro } \\
\text { asunto...] (1:3379-2:175) }\end{array}$ \\
\hline
\end{tabular}

Fuente: elaboración propia.

Finalmente, para una mejor comprensión de los resultados, la tabla 4 muestra datos como el orden dado en la Unidad Hermenéutica (UH) a cada orientación (documento o entrevista), el nombre del documento, la sigla establecida, la sigla designada para la presentación y el análisis de resultados, y un ejemplo de identificador numérico para las citas respectivas.

Para el caso del presente artículo se responde las preguntas: ¿cuáles son los tipos de contenidos escolares en los LCNEA (D1, D2 y P7), los ECN
(D3 y P8) y los DCN (D4, D5, D6 y P9) y a qué niveles de organización responden?

\section{Resultados.}

Los énfasis en los tipos de contenidos de las orientaciones, como se indica en la tabla 5, son: contenidos conceptuales y procedimentales, contenidos procedimentales, contenidos conceptuales, y contenidos actitudinales.

3. El identificador numérico de la cita está compuesto por un primer número que indica el número del documento en la UH y un segundo número referente al orden en el que se ha creado la cita. Entre corchetes se indica la cita. Los últimos números entre paréntesis señalan el párrafo de inicio y el fin de la cita. Así por ejemplo para 1:310 [El artículo 67 establece que...] (27:3470-27:3740) quiere decir que 1:310, es el documento P1 LCNEA, la cita 310, entre corchetes la frase con la que inicia la cita [El artículo 67 establece que...] y los números entre paréntesis (27:3470-27:3740) representan la selección de la unidad de información "El artículo 67 establece que "la educación formará al colombiano en el respeto a los derechos humanos, a la paz y a la democracia; y en la práctica del trabajo y la recreación, para el mejoramiento cultural, científico, tecnológico y para la protección del ambiente", de los Lineamientos Curriculares - Ciencias Naturales y Educación Ambiental LCNEA. 
En cuanto a los contenidos conceptuales y procedimentales, la mayoría se caracteriza por relacionar los contenidos conceptuales del conocimiento científico y las maneras como este se produce, también se encuentran los que integran el conocimiento de las ciencias con prácticas personales, sociales y ambientales, y en menor número aparecen los que se refieren a la relación de contenidos y prácticas de la vida cotidiana o contextual. Los documentos que mayor aporte hacen a esta subcategoría son los DCN (D5), luego los ECN (D3), y finalmente los LCNEA (D2).

Los contenidos procedimentales, que refieren a contenidos que tienen su base en las formas como se produce el conocimiento científico (los DCN), plantean una propuesta de habilidades científicas organizadas en tres ámbitos: investigación, representación y comunicación. También se encuentran contenidos de prácticas o comportamientos de índole personal y social, cuyas mayores contribuciones las hace el cuadro anexo a los LCNEA (D2), los ECN (D3) y los DCN (D4);

En cuanto a los contenidos conceptuales con relevancia en los conocimientos de las ciencias (procesos biológicos, físicos y químicos), unos se refieren a las relaciones CTS y otros con situaciones del orden social como la salud, el ambiente, las mayores contribuciones las hace el cuadro anexo a los LCNEA (D2, D1), los DCN (D5, D4) y, con pocas citas, los ECN (D3).

Sobre los contenidos actitudinales el mayor número se refiere a actitudes, prácticas y compromisos en el plano personal, a la relación con los otros, al cuidado del entorno y al asumir posturas frente a situaciones de las problemáticas ambientales. Los documentos que mayores aportes hacen son los ECN (D3), ya que la propuesta a lo largo de los conjuntos de grados plantean el entorno "Ciencia-Tecnología-Sociedad-CTS" y el eje "DesarroIlo compromisos personales y sociales"; luego el aporte de citas lo hace la tabla anexa de los LCNEA (D2), que propone el proceso "conocimiento en el mundo de la vida" $y$, finalmente, con un mínimo de citas los DCN (D5), específicamente a través de las evidencias de aprendizaje asociadas al enunciado de los DCN.

Para el caso de los niveles de organización, como se aprecia en la tabla 5, se presentaron los siguientes énfasis: conceptos o aprendizajes estructurantes, formulación de niveles de complejidad, procesos en la construcción de conocimientos o en la definición de macro contenidos de las ciencias naturales, y ejes articuladores.

Los conceptos o aprendizajes estructurantes se refieren a unidades básicas para el aprendizaje en el contexto escolar y para otros aprendizajes en la vida, que posibilitan además movilizar otros saberes, actitudes y prácticas; los mayores aportes a esta categoría los hacen los DCN (D5, D4), y con un número reducido de citas los LCNEA (D1). Es importante mencionar que la gran mayoría de estos aprendizajes estructurantes tiene como base el conocimiento científico.

La formulación de niveles de complejidad plantea un proceso espiral para el abordaje de los diferentes tipos de contenidos escolares; en todas las orientaciones se reconoce la necesidad de partir de un conocimiento cotidiano que se enriquece a través de los diferentes grados o conjuntos de grados, aunque no se pretende la "formación de científicos", la meta es el "desarrollo de pensamiento científico", cuyo referente epistemológico de mayor preponderancia es el conocimiento científico y las maneras de proceder de la comunidad científica, como ya se ha venido mencionando. Los documentos que hacen mayores aportes a esta subcategoría son LCNEA (D1, D2) y los ECN (D3), y para el caso de los DCN se hace explícita esta complejidad en la propuesta de progresiones de aprendizaje en el documento de mallas (D5).

En cuanto a los procesos en la construcción de conocimientos, o en la definición de macro contenidos de las ciencias naturales, se hace referencia a la articulación de diferentes tipos de contenidos, que implican complejidades diversas. Adicionalmente, para abordar esos contenidos se requiere de acciones de integración, relación, profundización, que generan redes o trayectorias que 
Tabla 5. Recurrencia de citas de las subcategorías de la macro categoría "contenidos escolares" (tipos y niveles de organización)-LCNEA-ECN-DCN

\begin{tabular}{lcccc}
\hline \multicolumn{1}{c}{ Subcategorías de la macro categoría “contenidos escolares” } & \multicolumn{2}{c}{ Orientaciones } & Total \\
\cline { 2 - 5 } A.1. Tipos de contenidos escolares & D1,2, P7 & D3, P8 & D4,5,6, P9 \\
\hline A.1.1. Contenidos conceptuales & 206 & 6 & 110 & 322 \\
\hline A.1.2. Contenidos procedimentales & 154 & 110 & 74 & 338 \\
\hline A.1.3. Contenidos actitudinales & 19 & 65 & 3 & 87 \\
\hline A.1.4. Contenidos conceptuales y procedimentales & 17 & 232 & 262 & 511 \\
\hline A.1.5. Contenidos conceptuales y actitudinales & 0 & 13 & 10 & 23 \\
\hline A.1.6. Contenidos procedimentales y actitudinales & 7 & 5 & 2 & 14 \\
\hline A.1.7. Contenidos conceptuales, procedimentales y actitudinales & 9 & 6 & 12 & 27 \\
\hline A.2. Niveles de organización de los contenidos escolares & & & & \\
A.2.1. Visión simple de organización & & & & \\
\hline A.2.1.1. Listado de contenidos (a modo de listado de temas) & 0 & 0 & 0 & 0 \\
\hline A.2.2. Visión compleja de organización & & & & \\
\hline $\begin{array}{l}\text { A.2.2.1. Procesos en la construcción de conocimientos o en la definición de } \\
\text { macro contenidos de las ciencias naturales }\end{array}$ & 38 & 31 & 0 & 69 \\
\hline A.2.2.2. Formulación de niveles de complejidad & 46 & 14 & 13 & 73 \\
\hline A.2.2.3. Ejes articuladores & 1 & 9 & 10 & 20 \\
\hline A.2.2.4. Proyectos pedagógicos & 5 & 0 & 0 & 5 \\
\hline A.2.2.5. Conceptos o aprendizajes estructurantes & 2 & 0 & 107 & 109 \\
\hline A.2.2.6. Mapa de relaciones & 0 & 0 & 3 \\
\hline
\end{tabular}

Fuente: elaboración propia.

tienen avances, regresos, idas, y que posibilitan la construcción de conocimiento y el desarrollo de pensamiento crítico, analítico y reflexivo. Los documentos que hacen el mayor aporte a este nivel de organización son de los LCNEA (D1, D2), al igual que los ECN (D3), desde la propuesta que hacen de estándares de competencias (4 o máximo 5) por conjunto de grado.

Sobre los ejes articuladores que permiten la organización de los diferentes tipos de contenidos relacionados con los conocimientos de las ciencias (procesos biológicos, procesos físicos y procesos químicos), al igual que las relaciones ciencia-tecnología y sociedad, los documentos que contribuyen con el mayor número de citas son los DCN, en el documento de la fundamentación teórica (D4), a partir del análisis de los ejes en los currículos internacionales y nacionales, que luego fueron insumo para la definición de los conceptos estructurantes, y en las mallas de aprendizaje (D6) como categorías organizadoras (entorno físico y entorno vivo) de los DCN. También los ECN (D3) con los tres ejes articuladores "Me aproximo al conocimiento como científico natural", "Manejo conocimientos propios de las ciencias naturales" y "Desarrollo compromisos personales y sociales".

\section{Conclusiones}

En cuanto al qué del conocimiento escolar en ciencias (tipos de contenidos escolares y niveles de organización) de las orientaciones curriculares, se concluye que en todas se encuentra una diversidad de contenidos conceptuales, procedimentales y actitudinales, pero la articulación entre los contenidos escolares se da con mayor énfasis entre 
los contenidos conceptuales y los procedimentales, aunque tienen diferentes referentes epistemológicos de base (cotidiano, contextual, cultural). Por su parte, el referente preponderante para este tipo de contenidos es el conocimiento científico y las actividades que se realizan en la comunidad científica; los contenidos actitudinales se presentan en relación con los contenidos conceptuales o con los procedimentales, pero en menor porcentaje. Estos contenidos hacen énfasis en valores, actitudes y compromisos personales, sociales y ambientales, y se identifica la siguiente tensión ¿la integración de los tipos de contenidos escolares está dada para lograr comprensiones más holísticas, o para la comprensión del conocimiento científico?

Frente a la diversidad de contenidos y las integraciones que se presentan, se reconoce en la $\mathrm{Hi}-$ pótesis de Progresión-Transición HdP-T un tránsito entre el nivel 2A (instruccional/cientificista-NIC) y el nivel 3 (integrador/transformador-NIT), lo anterior se debe a que principalmente los contenidos conceptuales y procedimentales tienen su base en las disciplinas científicas y las actividades que hace la comunidad científica para llevar a cabo las prácticas, para registrar los procedimientos, organizar información, estructurar los resultados y para socializarlos. Sin embargo, cuando los contenidos escolares se presentan de manera integral (conceptuales-actitudinales, conceptuales-procedimentales-actitudinales, o actitudinales-procedimentales) proponen un diálogo entre los conceptos de las ciencias con asuntos de la vida personal y social. Esto deja ver, por una parte, las relaciones entre los tipos de contenidos y las finalidades para el conocimiento escolar en ciencias (ej. comprender situaciones o fenómenos del contexto) y junto con las finalidades generales de la educación (ej. fin noveno de la educación: el desarrollo de la capacidad crítica, reflexiva y analítica que fortalezca el avance científico y tecnológico nacional orientado con prioridad al mejoramiento cultural y de la calidad de vida). Y, por otra parte, la relación con referentes epistemológicos como el conocimiento contextual y cultural, y el conocimiento social y ciudadano, entre otros.

En cuanto a los niveles de organización de los contenidos escolares, las orientaciones curriculares proponen una visión compleja de organización que estarían en función de tres elementos fundamentalmente: uno relacionado con la estructura de los contenidos escolares (ej. conceptos estructurantes), otro que se refiere a los niveles de complejidad a medida que se avanza de grado o conjunto de grado (ej. niveles de complejidad) y otro con las formas de abordar los contenidos que se proponen (ej. proyectos pedagógicos). Sin embargo, aquí emerge la pregunta: ¿esta visión compleja de organización de los contenidos escolares se establece para lograr la construcción de conocimientos científicos, o para una construcción de conocimientos en busca de una comprensión más holística y compleja del mundo?

Lo anteriormente expuesto se reflejó en los niveles de complejidad en la Hipótesis de Progresión-Transición HdP-T, en la que se encontró un énfasis en el nivel 3 (integrador/ transformador-NIT), porque a lo largo de los grados o los conjuntos de grado (lectura vertical) no solo se busca un proceso de construcción, sino que se propone la integración de contenidos por conjunto de grado, articulación entre ejes o procesos (lectura horizontal), o en el caso de los grados, progresiones de aprendizaje. Estas características de una visión compleja de organización se reconocen como una construcción pedagógica y didáctica propia que debería reflejarse en la propuesta de organización curricular y en las prácticas pedagógicas.

Finalmente, se podría concluir que en el marco del análisis de la macro categoría "contenidos escolares" (tipos y niveles de organización de los contenidos escolares) se analizan los tipos de contenidos escolares, los cuales están vinculados de manera directa con una visión disciplinar (nivel instruccional-cientificista). En cambio, para la organización de los contenidos escolares se propone una construcción pedagógica y didáctica propia (nivel integrador-transformador). Aspectos 
que consideramos relevantes para profundizar, a propósito de la consideración del conocimiento escolar, como conocimiento particular, a la luz de otras categorías analizadas (finalidades educativas, fuentes y criterios de selección de los contenidos escolares, referentes epistemológicos del conocimiento escolar y criterios de validación del conocimiento escolar), tal como se desarrolla en Cárdenas (2021).

\section{Referencias}

Bardín, L. (1996). El análisis de contenido. 2a ed. Madrid: Akal

Cárdenas, A. (2021). El conocimiento escolar en los lineamientos curriculares, estándares básicos de competencias y derechos básicos del aprendizaje para el área de Ciencias Naturales en Colombia: estudio de caso (Tesis Doctoral). Bogotá: Universidad Distrital Francisco José de Caldas

Chernobilsky, L. (2006). El uso de la computadora como auxiliar en el análisis de datos cualitativos. En I. Vasilachis de Gialdino (coord.), Estrategias de investigación cualitativa. Gedisa

Congreso de Colombia. (1994). Artículo 78 [Título IV]. Ley General de Educación. [Ley 115 de 1994]. https://www.mineducacion.gov.co/1621/articles-85906 archivo pdf.pdf

Congreso de Colombia. (1995). Ley 188 de 1995. Plan Nacional de Desarrollo e Inversiones 1995-1998. http://www.secretariasenado.gov.co/senado/basedoc/ley $0188 \quad 1995 . \mathrm{html}$

Departamento Nacional de Planeación. (s.f.). Bases del Plan Nacional de Desarrollo. 2014-2018. https:// colaboracion.dnp.gov.co/CDT/Prensa/PND\%20 2014-2018\%20Bases\%20Final.pdf

Flick, U. (2012). Introducción a la investigación cualitativa. Madrid: Morata

García, E. (1998). Hacia una teoría alternativa sobre los contenidos escolares. Sevilla: Díada

Goodson, I. (1991). La construcción social del currículum, posibilidades y ámbitos de investigación de la historia del currículum. Revista de Educación, 295, 7-37
Martínez, C. (2000). Las propuestas curriculares sobre el conocimiento escolar en el área de conocimiento del medio: dos estudios de caso en profesores de primaria (Tesis Doctoral). España: Universidad de Sevilla

Martínez, C. (2005). Las propuestas de conocimiento escolar en los inicios del aprendizaje de la química: un estudio de caso en las clases de ciencias en sexto grado de educación primaria. Centro de Investigaciones, Universidad del Tolima

Martínez, C. (2016). El conocimiento profesional de profesoras de ciencias de primaria sobre el conocimiento escolar: dos estudios de caso, en Aulas Vivas y Aulas Hospitalarias del Distrito Capital de Bogotá. Bogotá: Universidad Distrital Francisco José de Caldas

Martínez, C. (2017). Ser maestro de Ciencias. Productor de conocimiento profesional y de conocimiento escolar. Bogotá: Universidad Distrital Francisco José de Caldas

Martínez, C., Martínez, V. (2012). El conocimiento escolar y las hipótesis de progresión: algunos fundamentos y desarrollos. Nodos y Nudos, 32, 50-63

Martínez, C., Valbuena, E. (comps.) (2013). El conocimiento profesional de los profesores de ciencias sobre el conocimiento escolar. Resultados de Investigación. Doctorado Interinstitucional en Educación. Bogotá: Universidad Distrital Francisco José de Caldas

Martínez, C., Lopes, A., Cárdenas, A., Jirón, M. (2016). Las propuestas de conocimiento escolar en ciencias naturales en las orientaciones curriculares de la Secretaría de Educación de Bogotá (2007-2015). Proyecto de Investigación. Centro de Investigaciones y Desarrollo Científico. Universidad Distrital Francisco José de Caldas

Mason, J. (1996). Qualitative Researching. Sage Publications

Mauri, T. (1993). Los contenidos escolares. Aula de Innovación Educativa, 11, 57-60

MEN. (s.f.). Plan Sectorial 2002-2006. https://www.mineducacion.gov.co/1621/articles-85273_archivo_ pdf.pdf

MEN. (1998). Lineamientos Curriculares. Ciencias Naturales y Educación Ambiental. Ministerio de 
Educación Nacional. https://www.mineducacion. gov.co/1759/articles-89869_archivo_pdf5.pdf

MEN. (2006). Estándares Básicos de Competencias en Lenguaje, Matemáticas, Ciencias y Ciudadanas. Ministerio de Educación Nacional. https://www.mineducacion. gov.co/1621/articles-340021 recurso 1.pdf

MEN. (2010). Plan sectorial 2010-2014. Documento No 9. https://www.mineducacion.gov.co/1759/articles-293647_archivo_pdf_plansectorial.pdf

MEN. (2016). Derechos Básicos de Aprendizaje. Ministerio de Educación Nacional. http://aprende.colombiaaprende.edu.co/sites/default/files/naspublic/ DBA_C.Naturales.pdf

MEN. (2017). Mallas de aprendizaje. Ciencias Naturales y Educación Ambiental. Grado 5․ Documento para la implementación de los DBA. Bogotá. https://aprende.colombiaaprende.edu.co/ckfinder/ userfiles/files/NATURALES-GRADO-5 .pdf

MEN. (2017). Mallas de aprendizaje. Documento para la implementación de los DBA. Presentación general y por Áreas. Bogotá. https://aprende. colombiaaprende.edu.co/ckfinder/userfiles/files/ CARTILLA-INTRODUCTORIA .pdf
Merchán, F. J., García, F. (1997). El tratamiento de problemas de nuestro mundo en la enseñanza obligatoria. Aula de Innovación Educativa, 61, 9-12

Moraes, R. y Galiazzi, M. (2006). Análise textual discursiva: processo reconstrutivo de múltiplas faces. Ciência \& Educação, 12(1), 117-122

Senado de la Republica de Colombia. (2001). Inciso 5.5 Artículo 5 [Título II]. Ley 715 de 2001. https://www. mineducacion.gov.co/1621/articles-86098_archivo_pdf.pdf

Stake, R. E. (1999). Investigación con estudio de casos. Ediciones Morata

Universidad de Antioquia - MEN. (2016). Fundamentación teórica de los DBA. Producto Nro. 11: Documento con la fundamentación teórica de los DBA que indique justificación, antecedentes, referentes legales, teóricos, conceptuales que sustentan la propuesta. http://aprende.colombiaaprende.edu. co/ckfinder/userfiles/files/fundamentacioncienciasnaturales.pdf

Vasilachis, I. (2006). La investigación cualitativa. En I. Vasilachis de Gialdino (coord.). Estrategias de investigación cualitativa. Gedisa 